\title{
Emergência e desenvolvimento inicial de porta-enxertos de citros no semiárido do Ceará, Brasil
}

\author{
Kassio Ewerton Santos Sombra ${ }^{1}$, Alexandre Caique Costa e Silva ${ }^{2}$, \\ Francisco Leandro Costa Loureiro ${ }^{3}$, Henrique Maia de Andrade ${ }^{2}$, Debora Costa Bastos ${ }^{4} \&$ \\ Cleilson do Nascimento Uchôa ${ }^{2}$
}

\begin{abstract}
RESUMO
A baixa diversidade de porta-enxertos consolidou-se como uma das principais vulnerabilidades da citricultura brasileira, predominando por exemplo, seleções de limão Cravo (Citrus limonia Osbeck.) (Rutaceae) de alta rusticidade e compatíveis com as principais variedades-copa. Porém, a uniformização dos planteis cítricos eleva o risco diante de estresses bióticos e/ou abióticos, necessitando de estudos que fomentem a diversificação, principalmente com porta-enxertos resistentes ou tolerantes. Diante disto, o trabalho teve objetivo de avaliar e caracterizar a emergência e o desenvolvimento inicial de citrandarins Riverside, San Diego e Índio (Citrus sunki Hayata hort. ex Tanaka $\times$ Poncirus trifoliata L. Raf.), além de tangerineira Sunki Tropical (C. sunki) e limão Cravo (C. limonia). O limoeiro Cravo apresentou a menor taxa final de emergência, diferindo estatisticamente da tangerineira Sunki Tropical e dos citrandarins Riverside e San Diego, com apenas 18,5\% de plântulas emergidas aos 90 dias após a semeadura, porém, não diferiu do citrandarin Indio, que obteve 58,5\% de emergência no mesmo período. Os citrandarins Riverside e San Diego demonstraram maior precocidade e uniformidade na emergência e no desenvolvimento inicial das plântulas, não diferindo estatisticamente da tangerineira Sunki Tropical, mostrando-se alternativas viáveis ao limoeiro Cravo Santa Cruz, que obteve os menores resultados em todas as variáveis analisadas, e diante da sua susceptibilidade a estresses bióticos e abióticos de importância econômica, tem sido menos recomendado e menos empregado em novas implantações.
\end{abstract}

Termos de indexação: citricultura, diversificação, enxertia, mudas, viveiro.

\section{Emergence and initial development of citrus rootstocks in the semi-arid of Ceará, Brazil}

\section{SUMMARY}

The low diversity of rootstocks has been consolidated as one of main vulnerabilities of the Brazilian citrus crop, predominating, for example, selections of Rangpur lemon (Citrus limonia Osbeck.) (Rutaceae) of high rusticity and compatible with the main varieties-canopy. However,

\footnotetext{
${ }^{1}$ Departamento de Fitotecnia, Universidade Federal do Ceará - UFC, Fortaleza, CE, Brasil

${ }^{2}$ Departamento de Agronomia, Instituto Federal de Educação, Ciência e Tecnologia do Ceará - IFCE, Fortaleza, CE, Brasil

${ }^{3}$ Departamento de Fitotecnia, Universidade Federal Rural do Semiárido - UFERSA, Mossoró, RN, Brasil

${ }^{4}$ Empresa Brasileira de Pesquisa Agropecuária, Petrolina, PE, Brasil

Autor correspondente: Kássio Ewerton Santos Sombra, Departamento de Fitotecnia, Universidade Federal do Ceará - UFC, Avenida Mister Hull, 2977, Bloco 805, Campus do Pici, CEP 60356001, Fortaleza, CE, Brasil. E-mail: kassiosombra@gmail.com
} 
the uniformity of citrus plants increases the risk of biotic and/or abiotic stresses, requiring studies that encourage diversification, especially with resistant or tolerant rootstocks. The objective of this work was to evaluate and characterize the emergence and inital development of Riverside, San Diego e Indio citrandarins (Citrus sunki Hayata hort. ex Tanaka x Poncirus trifoliata L. Raf.), As well as tropical Sunki tangerine (C. sunki) and Rangpur lemon (C. limonia). Rangpur lemon presented the lowest final emergence rate, statistically differing from Sunki Tropical tangerine and Riverside and San Diego citrandarins, with only 18.5\% of seedlings emerged at 90 days after sowing, but did not differ from citrandarin Indio, which obtained an emergency $58.5 \%$ in the same period. The Riverside and San Diego citrandarins demonstrated greater precocity and uniformity in the emergence and initial development of the seedlings, not statistically differing from the Sunki Tropical tangerine, showing viable alternatives to the Rangpur lemon Santa Cruz, which obtained the lowest results in all analyzed variables, and because of its susceptibility to biotic and abiotic stresses of economic importance, has been less recommended and less used in new implantations.

Index terms: citriculture, diversification, grafting, seedlings, nursery.

\section{INTRODUÇÃO}

A baixa diversidade de porta-enxertos consolidou-se como uma das principais vulnerabilidades da citricultura brasileira, predominando o uso de seleções de limão Cravo (Citrus limonia Osbeck.) (Rutaceae), como constata-se, por exemplo, em 40,26\% das plantas cítricas de São Paulo, principal polo citrícola nacional (CDA, 2018).

O limão Cravo (C. limonia) tornou-se conhecido por sua rusticidade e adaptação a diferentes condições edafoclimáticas, destacando-se por apresentar resistência à seca, compatibilidade com as principais variedades-copa nacionais, como por exemplo, as laranjas Pêra, Valência e Natal, as quais o limão Cravo pode inferir precocidade e produtividade, além de apresentar tolerância ao vírus causador da tristeza (Citrus tristeza vírus, CTV) (Soares Filho et al., 2003a; Barbosa \& Rodrigues, 2015).

Porém, as seleções de limão Cravo apresentam susceptibilidade aos fungos causadores da gomose, Phytophthora citrophthora (R.E. Sm. \& E.H. Sm.) Leonian e Phytophthora nicotianae var parasitica (Dastur) G.M. Waterh (Oomycetes, Pythiales), e da verrugose, Elsinoë fawcettii Bitanc. \& Jenkins (Dothideomycetes, Myriangiales), assim como, susceptibilidade ao declínio e a morte súbita dos citros (MSC), e intolerância aos viróides da exocorte (Citrus exocortis viroid, CEVd) e xiloporose (Hop stunt viroid, HSVd) (Soares Filho et al., 2003a; Eiras et al., 2009; Najar et al., 2018).

Diante disto, tem crescido o número de estudos sobre porta-enxertos, alternativos ao limão Cravo, tolerantes ou resistentes aos principais estresses bióticos e abióticos, como por exemplo, os citrandarins Índio, Riverside e San Diego (Citrus sunki Hayata hort. ex Tanaka $\times$ Poncirus trifoliata L. Raf.) (Passos et al., 2007; Passos, 2012; Pompeu Junior
\& Blumer, 2014; Carvalho et al., 2016), favorecendo a diversificação, e consequentemente, a segurança nos pomares e polos citrícolas (Rodrigues et al., 2012).

Os citrandarins são híbridos de tangerineira Sunki (C. sunki) com Poncirus trifoliata (P. trifoliata), obtidos pelo Dr. Joe Randolph Furr. na Califórnia e selecionados pelo Programa de Melhoramento Genético de Citros PMG Citros da Embrapa Mandioca e Fruticultura, por apresentarem, entre outras vantagens, alta adaptabilidade às condições tropicais, compatibilidade com a maioria das variedades-copa de laranjeiras, tangerineiras e limeiras ácidas, equiparando-se a porta-enxertos tradicionais como o limoeiro Cravo, além de resistência aos fungos causadores da gomose (Phytophthora spp.) e a morte súbita dos citros (Passos et al., 2011a; Passos et al, 2011b; Passos et al., 2011c; Passos, 2012; Carvalho et al., 2016).

Os porta-enxertos são propagados, quase que exclusivamente, por sementes (via seminífera), onde destaca-se a importância dos potenciais de germinação, e consequentemente, de emergência, para o desenvolvimento inicial das plântulas, antecipando ou retardando a realização da enxertia (borbulhia) da variedade-copa, em alguns casos atingindo até $30 \%$ do tempo total de produção da muda cítrica, variando em função da precocidade e homogeneidade das sementes (Fochesato et al., 2006; Rodrigues et al., 2012; Cunha Sobrinho et al., 2013; Soares et al., 2015).

Diante disto, o trabalho teve objetivo de avaliar e comparar a emergência e o desenvolvimento inicial de porta-enxertos cítricos com tolerância ou resistência a estresses bióticos e/ou abióticos. 


\section{MATERIAL E MÉTODOS}

O trabalho foi desenvolvido entre julho e novembro de 2016 em ambiente protegido com área de $220,5 \mathrm{~m}^{2}(6,3 \mathrm{~m} \times 35 \mathrm{~m})$, na Unidade de Ensino, Pesquisa e Extensão - UEPE do Instituto Federal de Educação, Ciência e Tecnologia do Ceará - IFCE, localizada na Chapada do Apodi, a latitude $5^{\circ} 10^{\prime} 56.82$ " S e longitude $38^{\circ} 0^{\prime} 46.33^{\prime}$ ' O, a uma altitude aproximada de $146 \mathrm{~m}$, Limoeiro do Norte, CE, Brasil (Figura 1).

A clima da região é classificado como seco e muito quente, do tipo BSw'h' (Köppen) (Alvares et al., 2013), com duas estações climáticas bem definidas, uma seca, que vai geralmente entre junho e janeiro; e uma chuvosa, de fevereiro a maio. A precipitação média anual da região é de $772 \mathrm{~mm}$, com temperatura média anual de $28,5^{\circ} \mathrm{C}$, oscilando entre $22^{\circ} \mathrm{C}$ de temperatura mínima e $35^{\circ} \mathrm{C}$ de máxima, resultado de uma insolação de $3.030 \mathrm{~h}$ por ano, baixa umidade relativa (62\%) e alta evapotranspiração (3.215 mm) (ADECE, 2011).

O ambiente protegido é do tipo viveiro telado, localiza-se com orientação Leste-Oeste, com cobertura de polietileno de baixa densidade (PEBD), transparente, e possui laterais revestidas com tela anti-afídeos (40 Mesh).

O delineamento experimental foi inteiramente casualizado (DIC), com cinco tratamentos (porta-enxertos), oito repetições e parcela útil de 25 sementes (Brasil, 2009; Banzatto \& Kronka, 2013).

As sementes dos porta-enxertos foram disponibilizadas através do Banco Ativo de Germoplasma de Citros da Embrapa - Mandioca e Fruticultura Tropical, situada em Cruz das
Almas, BA, Brasil. As sementes foram obtidas a partir de frutos de limão Cravo Santa Cruz (C. limonia); de citrandarins Riverside, San Diego e Índio (C. sunki x . trifoliata); e de tangerineira Sunki Tropical (C. sunki).

Os frutos foram colhidos manualmente ou com ganchos, em seguida, abertos com canivete para retirada cuidadosa das sementes. As sementes foram colocadas em bandeja plástica e lavadas com solução de água e cal virgem, na proporção de duas colheres de sopa de cal para 1 litro de água, retirando-se a mucilagem. Posteriormente, as sementes foram mergulhadas em solução de hipoclorito de sódio à $0,25 \%$ por 5 minutos para eliminar o excesso de contaminantes, e por último, lavadas com água potável para retirar o excesso de hipoclorito, permanecendo em repouso para secagem (Carvalho et al., 2016).

A determinação do teor de umidade (\%) das sementes foi realizada com base no peso úmido através do método de estufa com circulação forçada de ar a $45 \pm 3{ }^{\circ} \mathrm{C}$ (Brasil, 2009). Realizando-se a semeadura em tubetes cônicos de polietileno preto $\left(180 \mathrm{~cm}^{3}\right)$, vazados na parte basal, fixados em bancadas de madeira a 1,2 $\mathrm{m}$ do solo, semeando-se uma semente por tubete, na posição horizontal a uma profundidade de $1,5 \mathrm{~cm}$ a $2 \mathrm{~cm}$ (Araújo et al., 2015). Utilizou-se substrato orgânico preparado com areia quartzosa, terra de formigueiro e composto orgânico (compostagem), numa proporção de 1:1:1, peneirado e homogeneizado. As irrigações foram realizadas por microaspersão, aplicando-se uma lâmina média diária de $5 \mathrm{~mm}$.

Analisaram-se as porcentagens de emergência (PE), do vigésimo ao sexagésimo dia após a semeadura (DAS),

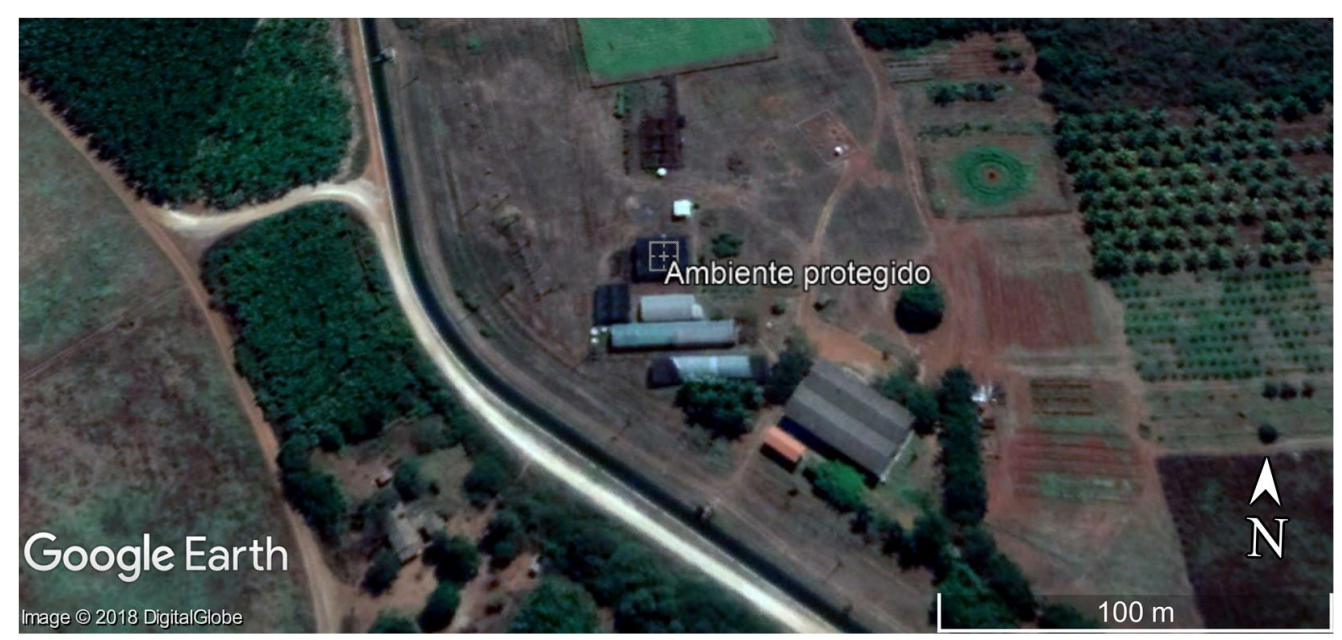

Figura 1. Ambiente Protegido da Unidade de Ensino, Pesquisa e Extensão - UEPE, Limoeiro do Norte - CE, Brasil. Fonte: Google Earth Pro 7.1.5.1557. Data da imagem: 18/07/2018. 
com intervalo de 2 dias entre leituras, realizando-se inspeções visuais e anotando-se as emergências sempre que a plúmula se encontrava ao menos $2 \mathrm{~mm}$ acima do substrato, os valores foram utilizados para cálculo do índice de velocidade de emergência (IVE), conforme formula a seguir (Nakagawa, 1994):

$\mathrm{IVE}=\mathrm{E} 1 / \mathrm{N} 1+\mathrm{E} 2 / \mathrm{N} 2+\mathrm{E} 3 / \mathrm{N} 3+\mathrm{E} 4 / \mathrm{N} 4 \ldots+\mathrm{En} / \mathrm{Nn}$ (Equação 1)

Onde: E1, E2, E3 e En são os números de plântulas emergidas na primeira, segunda, terceira, até a última leitura; N1, N2, N3 e Nn são os números de dias transcorridos desde a semeadura para a primeira, segunda, terceira, até a última leitura.

Aos 60 DAS determinaram-se as taxas finais de emergência (TFE) e poliembrionia (TFP) (Rodrigues et al., 2012; Rodrigues et al., 2015).

Realizaram-se avaliações biometrias para determinar o desenvolvimento das plântulas de porta-enxertos aos 30, 60 e 90 DAS, mensurando-se altura (h), medida do colo ao ápice, diâmetro da plântula (D) e diâmetro do caule $\left(\mathrm{d}^{2}\right)$, medido ao nível do colo, utilizando paquímetro digital (Rodrigues et al., 2012; Rodrigues et al., 2015).
Realizou-se análise de regressão $(\mathrm{p} \leq 0,01)$ para porcentagem de emergência (PE) em função do tempo para cada tratamento (porta-enxertos). Os dados obtidos, para todas as variáveis, foram submetidos à análise de variância (Anova), e nos casos de diferença significativa, compararam-se as médias pelo teste de Tukey $(\mathrm{p} \leq 0,05)$, utilizando-se software estatístico $\operatorname{SISVAR}^{\circledR}$ (Ferreira, 2014).

\section{RESULTADOS E DISCUSSÃO}

A análise de variância (Anova) permitiu constatar diferença significativa entre os tratamentos (porta-enxertos) para todas as variáveis de emergência e desenvolvimento analisadas, como é possível observar no resumo da Anova contido na Tabela 1.

O teor de umidade (\%) das sementes de limão Cravo Santa Cruz (C. limonia) diferiu estatisticamente das sementes dos citrandarins $(C$. sunki $\times P$. trifoliata $) \mathrm{e}$ da tangerineira Sunki Tropical (C. sunki), atingindo apenas $8,12 \%$ (Tabela 2 ), sendo que os procedimentos empregados na colheita, transporte e armazenamento,

Tabela 1. Resumo da análise de variância (Anova) para teor de umidade (U\%) nas sementes, taxas finais de emergência (TFE) e de poliembrionia (TFP), índice de velocidade de emergência (IVE), altura (H) e diâmetro das plântulas (D) aos 90 dias após a semeadura dos porta-enxertos, Limoeiro do Norte - CE, Brasil, 2018

\begin{tabular}{ccrrrrrr}
\hline \multirow{2}{*}{ FV } & \multirow{2}{*}{ GL } & \multicolumn{7}{c}{ Quadrados médios } \\
\cline { 3 - 7 } & & \multicolumn{1}{c}{ U\% } & \multicolumn{1}{c}{ TFE } & \multicolumn{1}{c}{ TFP } & \multicolumn{1}{c}{ IVE } & \multicolumn{1}{c}{ D } \\
\hline Porta-enxertos & 4 & $15,7^{*}$ & $4660,4^{*}$ & $810,8^{*}$ & $107,6^{*}$ & $46,3^{*}$ & $437,1^{*}$ \\
Resíduo & \multirow{2}{*}{35} & 0,79 & 923,58 & 9,05 & 21,68 & 6,97 & 146,32 \\
Média geral & & 10,02 & 58,35 & 10,30 & 8,40 & 14,75 & 70,27 \\
CV (\%) & 8,90 & 52,08 & 29,22 & 55,44 & 17,91 & 17,21 \\
\hline
\end{tabular}

$\mathrm{FV}=$ fonte de variação. $\mathrm{GL}=$ grau de liberdade. * significativo ao nível de $1 \%$ de probabilidade $(\mathrm{p} \leq 0,01)$.

Tabela 2. Teor de umidade nas sementes (\%), taxas finais de emergência (TFE) e de poliembrionia (TFP), além do índice de velocidade de emergência (IVE) aos 90 dias após a semeadura, Limoeiro do Norte, Ceará - Brasil, 2018

\begin{tabular}{ccccc}
\hline Porta-enxertos & Umidade $(\%)$ & TFE $(\%)$ & TFP $(\%)$ & IVE \\
\hline Limão Cravo Santa Cruz & $8,12 \mathrm{~b}$ & $18,50 \mathrm{~b}$ & $6,25 \mathrm{bc}$ & $2,25 \mathrm{~b}$ \\
Citrandarin Riverside & $10,75 \mathrm{a}$ & $64,25 \mathrm{a}$ & $7,50 \mathrm{~b}$ & $9,75 \mathrm{a}$ \\
Citrandarin San Diego & $11,50 \mathrm{a}$ & $83,50 \mathrm{a}$ & $28,00 \mathrm{a}$ & $11,50 \mathrm{a}$ \\
Citrandarin Indio & $10,75 \mathrm{a}$ & $58,50 \mathrm{ab}$ & $7,00 \mathrm{bc}$ & $8,00 \mathrm{ab}$ \\
Tangerina Sunki Tropical & $9,0 \mathrm{a}$ & $67,00 \mathrm{a}$ & $2,75 \mathrm{c}$ & $10,50 \mathrm{a}$ \\
C. V. (\%) & 8,90 & 52,08 & 29,22 & 55,44 \\
Valor F & $19,807^{*}$ & $5,046^{*}$ & $89,526^{*}$ & $4,964^{*}$ \\
Valor-p & 0,0000 & 0,0026 & 0,0000 & 0,0028 \\
\hline
\end{tabular}

As médias seguidas pela mesma letra não diferem estatisticamente entre si. *significativo ao nível de $1 \%$ de probabilidade $(\mathrm{p}<0,01)$. 
foram similares para as sementes dos cinco porta-enxertos (Demba, 2013). Entretanto, os valores do limão Cravo Santa Cruz (C. limonia) permaneceram acima dos $8,0 \%$ propostos por Montenegro \& Salibe (1960) como grau de umidade crítico, e aproximaram-se dos 8,01\% obtidos por Struiving et al. (2013), porém, foram inferiores aos $10,38 \%$ de umidade obtidos por Siqueira et al. (2002).

A umidade é uma das principais características para manutenção da qualidade fisiológica das sementes, sendo que variações na tolerância a secagem de sementes de porta-enxertos de citros, classificadas como intermediárias ou recalcitrantes (King \& Roberts, 1979; Hong \& Ellis, 1995), podem associar-se a diversos fatores, como por exemplo: a espécie, a temperatura, o tipo de embalagem e o tempo de armazenagem (Carvalho et al., 2002; Demba, 2013; Struiving et al., 2013). Sendo que teores de umidade mais elevados podem proporcionar maior vigor, e consequentemente, rapidez na emergência e no desenvolvimento inicial, permitindo ao porta-enxerto uma vantagem diante de possíveis estresses bióticos ou abióticos, quando comparado com um de menor vigor (Carvalho et al. 2002; Giuliani et al., 2014).

Os menores valores de umidade, possivelmente, inferiram ao limoeiro Cravo Santa Cruz (C. limonia) a menor taxa final de emergência (TFE), diferindo estatisticamente da tangerineira Sunki Tropical (C. sunki) e dos citrandarins Riverside e San Diego (C. sunki $\times$ P. trifoliata), com apenas $18,5 \%$ de plântulas emergidas aos 90 dias após a semeadura, porém, não diferiu do citrandarin Indio (C. sunki $\times$ P. trifoliata), que obteve $58,5 \%$ de plântulas emergidas no mesmo período (Tabela 2). Sombra et al. (2015) obtiveram valores de TFE superiores, registrando $90 \%$, 98\% e 92\% de plântulas dos citrandarin Riverside, San Diego e Indio (C. sunki x P. trifoliata) emergidas, respectivamente, aos 90 dias após a semeadura, enquanto o limoeiro Cravo Santa Cruz (C. limonia) obteve apenas $20 \%$ de plântulas emergidas no mesmo período.

O citrandarin San Diego (C. sunki $\times$ P. trifoliata $)$ apresentou a maior taxa final de poliembrionia (TFP), diferindo dos demais porta-enxertos, com $28 \%$ de poliembrionia aos 90 dias após a semeadura, enquanto a tangerineira Sunki Tropical (C. sunki) apresentou a menor taxa com 2,75\% de poliembrionia. Rodrigues et al. (2015) obtiveram $97,5 \%$ de taxa de poliembrionia para o citrandarim San Diego, diferindo e superando os valores do presente estudo (Tabela 2).

A poliembrionia possui enorme importância na produção de mudas cítricas, sendo que a grande maioria das variedades produz sementes poliembriônicas derivadas do tecido nucelar do ovário circundante ao saco embrionário (Soares Filho et al., 2003b; Andrade et al., 2007; Alesa et al., 2010). Por um lado, menores taxas de poliembrionia, e consequentemente, um menor número de embriões por semente, favorece o aumento do tamanho e a taxa de germinação do embrião zigótico (Soares-Filho et al., 2000). Por outro lado, quanto maior a taxa de poliembrionia, maiores são as chances de se obter plantas de origem nucelar (clones da planta-matriz), característica extremamente desejável na multiplicação comercial de porta-enxertos (Duarte et al., 2013).

Os citrandarins Riverside e San Diego (C. sunki $\times$ P. trifoliata) não diferiram da tangerineira Sunki Tropical (C. sunki) quanto ao índice de velocidade de emergência (IVE) (Tabela 2), registrando-se valores similares aos obtidos por Rodrigues et al. (2015), que obtiveram índices de 9,41 para o citrandarin Riverside e 8,32 para a tangerineira Sunki Tropical. Enquanto o menor IVE foi obtido pelo limoeiro Cravo Santa Cruz (C. limonia), com apenas 2,21, não diferindo estatisticamente do citrandarin Indio (C. sunki $\times$ P. trifoliata), que obteve IVE de 8,00, valor igual ao obtido por Rodrigues et al. (2015) avaliando o limoeiro Cravo (C. limonia).

A correlação entre o índice de velocidade de emergência e o tempo foi linear positiva $\left(\mathrm{R}^{2}>80 \%\right)$ para as sementes dos cinco porta-enxertos, indicando que o tempo, influenciou o percentual de plântulas emergidas (Figura 2). Porém, ao comparar as cinco curvas de emergência constata-se que o limoeiro Cravo Santa Cruz (C. limonia) diferiu, apresentando um subdesenvolvimento, quando comparado aos demais.

Os citrandarins Riverside, San Diego e Indio (C. sunki $\times$ P. trifoliata), assim como a tangerineira Sunki Tropical (C. sunki), demonstraram maior precocidade na emergência, e consequentemente, a maior velocidade refletiu diretamente no desenvolvimento vegetativo das plântulas, como constata-se, por exemplo, nos valores obtidos pelo citrandarin San Diego, que aos 90 dias apresentava altura média de 18,21 cm (Tabela 3) e diâmetro médio de 75,4 mm (Tabela 4), com espessura de caule de $2,4 \mathrm{~mm}$ em média (Tabela 5), estatisticamente superiores aos obtidos pelo limoeiro Cravo Santa Cruz (C. limonia) "(Figura 2).

Rodrigues et al. (2015) obtiveram valores inferiores para o desenvolvimento vegetativo de plântulas de citrandarins Riverside, San Diego e Indio (C. sunki $\times$ P. trifoliata), registrando-se aos 124 após a semeadura plântulas com altura média de $8,94 \mathrm{~cm}$ e diâmetro médio de caule de $2,33 \mathrm{~mm}$, indicando que diversos fatores, além do 
Tabela 3. Altura $(\mathrm{h} / \mathrm{cm})$ das plântulas dos porta-enxertos aos 30, 60 e 90 dias após a semeadura, Limoeiro do Norte, Ceará - Brasil, 2018

\begin{tabular}{cccc}
\hline Porta-enxertos & 30 dias & 60 dias & 90 dias \\
\hline Limão Cravo Santa Cruz & $3,50 \mathrm{~b}$ & $7,25 \mathrm{c}$ & $12,12 \mathrm{~b}$ \\
Citrandarin Riverside & $4,50 \mathrm{ab}$ & $9,00 \mathrm{bc}$ & $15,25 \mathrm{ab}$ \\
Citrandarin San Diego & $5,00 \mathrm{a}$ & $11,62 \mathrm{a}$ & $18,25 \mathrm{a}$ \\
Citrandarin Indio & $4,87 \mathrm{a}$ & $10,00 \mathrm{ab}$ & $15,25 \mathrm{ab}$ \\
Tangerina Sunki Tropical & $3,37 \mathrm{~b}$ & $7,50 \mathrm{c}$ & $12,87 \mathrm{~b}$ \\
C. V. (\%) & 20,57 & 17,21 & 17,91 \\
Valor F & $6,133^{*}$ & $10,802^{*}$ & $6,636^{*}$ \\
Valor-p & 0,0008 & 0,0000 & 0,0004 \\
\hline
\end{tabular}

As médias seguidas pela mesma letra não diferem estatisticamente entre si. *significativo ao nível de $1 \%$ de probabilidade $(\mathrm{p}<0,01)$.

Tabela 4. Diâmetro $(\mathrm{D} / \mathrm{mm})$ das plântulas dos porta-enxertos aos 30, 60 e 90 dias após a semeadura, Limoeiro do Norte, Ceará - Brasil, 2018

\begin{tabular}{cccc}
\hline Porta-enxertos & 30 dias & 60 dias & 90 dias \\
\hline Limão Cravo Santa Cruz & $32,00 \mathrm{~b}$ & $62,87 \mathrm{a}$ & $57,62 \mathrm{~b}$ \\
Citrandarin Riverside & $37,62 \mathrm{~b}$ & $69,00 \mathrm{a}$ & $69,87 \mathrm{ab}$ \\
Citrandarin San Diego & $43,12 \mathrm{a}$ & $66,25 \mathrm{a}$ & $75,62 \mathrm{a}$ \\
Citrandarin Indio & $45,12 \mathrm{a}$ & $71,50 \mathrm{a}$ & $73,87 \mathrm{ab}$ \\
Tangerina Sunki Tropical & $33,87 \mathrm{~b}$ & $72,00 \mathrm{a}$ & $74,37 \mathrm{ab}$ \\
C. V. (\%) & 24,50 & 15,85 & 17,21 \\
Valor F & $2,935^{*}$ & $0,990 \mathrm{~ns}$ & $2,988^{*}$ \\
Valor-p & 0,0342 & 0,4260 & 0,319 \\
\hline
\end{tabular}

As médias seguidas pela mesma letra não diferem estatisticamente entre si. *significativo ao nível de 5\% de probabilidade $(\mathrm{p}<0,05) .{ }^{\text {ns }}$ não significativo.

Tabela 5. Diâmetro do caule $\left(\mathrm{d}^{2} / \mathrm{mm}\right)$ das plântulas de porta-enxertos aos 90 dias após a semeadura, Limoeiro do Norte, Ceará - Brasil, 2018

\begin{tabular}{cc}
\hline Tratamento & Diâmetro do caule $(\mathrm{mm})$ \\
\hline Limão Cravo Santa Cruz & $1,87 \mathrm{~b}$ \\
Citrandarin Riverside & $2,00 \mathrm{ab}$ \\
Citrandarin San Diego & $2,37 \mathrm{a}$ \\
Citrandarin Indio & $2,00 \mathrm{ab}$ \\
Tangerina Sunki Tropical & $1,87 \mathrm{~b}$ \\
C. V. (\%) & 15,89 \\
Valor F & $3,259^{*}$ \\
Valor-p & 0,0226 \\
\hline
\end{tabular}

As médias seguidas pela mesma letra não diferem estatisticamente entre si. * significativo ao nível de 5\% de probabilidade $(\mathrm{p}<0,05)$.

potencial genotípico, podem contribuir diretamente com o desenvolvimento inicial dos porta-enxertos, como por exemplo, as condições ambientais do local de estudo, como fotoperíodo, temperatura e radiação solar, assim como, a composição físico-química dos substratos, que devem ser preparados para atender as exigências nutricionais das plântulas, permitindo o desenvolvimento em altura e diâmetro, o que pode acelerar a etapa de obtenção dos porta-enxertos, e consequentemente, reduzir o tempo para realização da enxertia (borbulhia) da variedade-copa (Pompeu Junior \& Blumer, 2014; Sombra et al., 2015; Rodrigues et al., 2015; Simonetti, 2015).

Os citrandarins Riverside, San Diego e Indio (C. sunki $\times$ P. trifoliata), e a tangerineira Sunki Tropical (C. sunki), apresentaram emergência e desenvolvimento inicial superiores aos do limão Cravo Santa Cruz (C. limonia), reforçando o potencial de uso dos genótipos como porta-enxertos na produção de mudas cítricas, já motivado pelas características de resistência e/ou tolerância que os citrandarins e a tangerineira Sunki possuem para estresses bióticos e abióticos de importância econômica, como por exemplo, resistência a gomose (Phytophthora spp.) (Passos et al., 2011a; Passos et al., 2011b; Passos et al., 2011c; Passos, 2012; Rodrigues et al., 2015; Carvalho et al., 2016). 


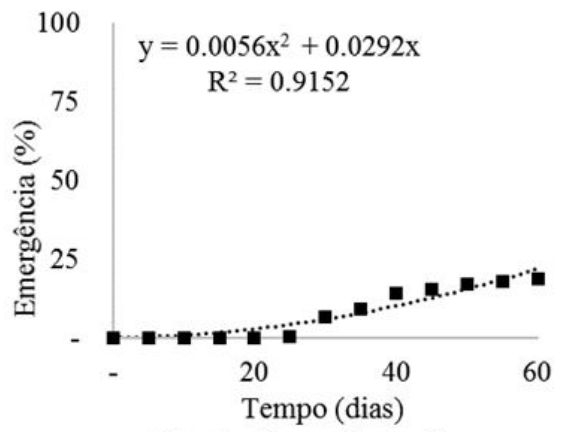

- Limão Cravo Santa Cruz

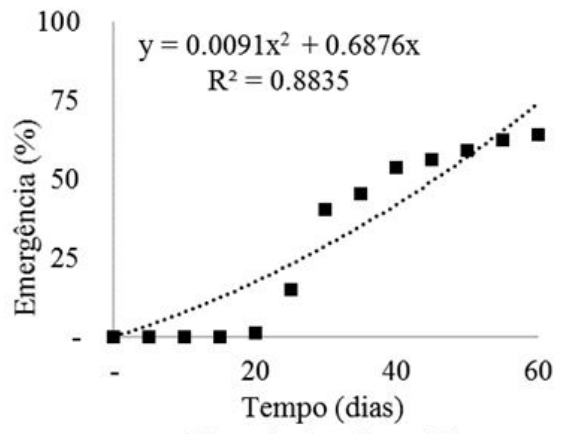

- Citrandarin Riverside

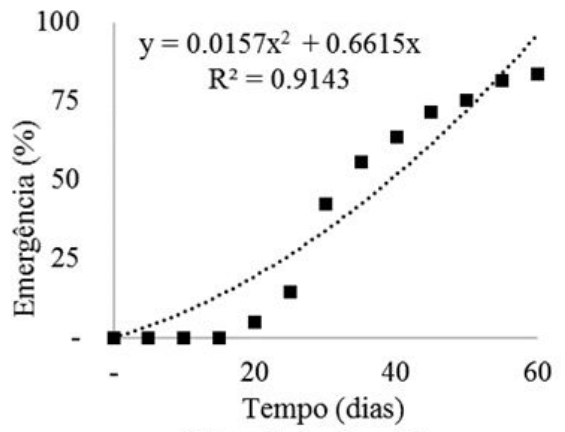

- Citrandarin San Diego

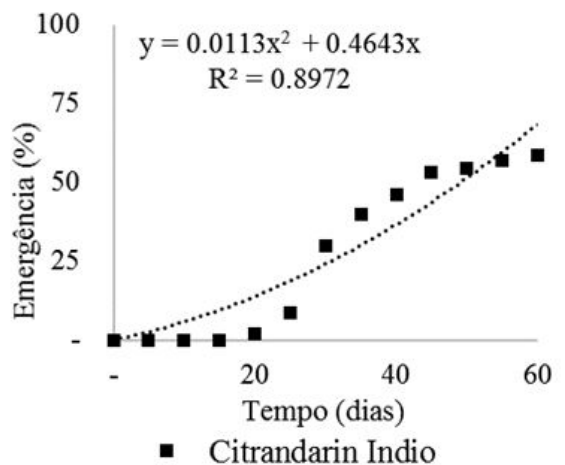

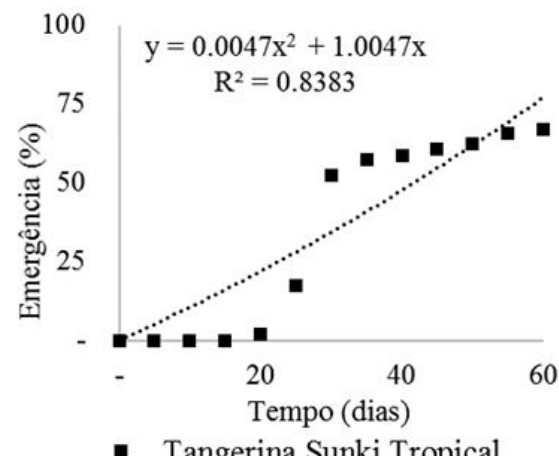

Figura 2. Curvas de emergências, equações de regressão e coeficientes de determinação $\left(\mathrm{R}^{2}\right)$ de limoeiro Cravo Santa Cruz (C. limonia), citrandarins Riverside, San Diego e Indio (C. sunki $\times$ P. trifoliata), e de tangerineira Sunki Tropical (C. sunki) em 60 dias após a semeadura, Limoeiro do Norte - CE, Brasil.

\section{CONCLUSÕES}

Os citrandarins Riverside e San Diego (C. sunki $\times$ P. trifoliata) demonstraram maior precocidade e uniformidade na emergência e no desenvolvimento inicial das plântulas, não diferindo estatisticamente da tangerineira Sunki Tropical (C. sunki), mostrando-se alternativas viáveis ao limoeiro Cravo Santa Cruz (C. limonia), que obteve os menores resultados em todas as variáveis analisadas, e diante da sua susceptibilidade a estresses bióticos e abióticos de importância econômica, tem sido menos recomendado e menos empregado em novas implantações.

\section{AGRADECIMENTOS}

Ao Conselho Nacional de Desenvolvimento Científico e Tecnológico (CNPq) pela concessão de bolsas, à Embrapa Mandioca e Fruticultura, à Embrapa Semiárido e à Secretaria de Agricultura de Russas (SEAGRI). 


\section{REFERÊNCIAS}

Agência de Desenvolvimento do Estado do Ceará ADECE. (2011). Perímetros públicos irrigados do Ceará. (20 p). Fortaleza: Governo do Estado do Ceará.

Alesa, P., Juárez, J., Ollitrault, P., \& Navarro, L. (2010). Polyembryony in non-apomictic citrus genotypes. Annals of Botany, 106(4), 533-545.

Alvares, C. A., Stape, J. L., Sentelhas, P. C., Moraes, G., Leonardo, J., \& Sparovek, G. (2013). Köppen's climate classification map for Brazil. Meteorologische Zeitschrift, 22(6), 711-728.

Andrade, R. A., Martins, A. B. G., Lemos, E. G. M., Luz, F. J. F., \& Silva, M. T. H. (2007). Detecção de polimorfismo em porta-enxertos para citros. Revista Brasileira de Fruticultura, 29(2), 345-349.

Araújo, R. A., Siqueira, D. L., Martinez, C. A., \& Fernandes, A. R. (2015). Características biométricas, índice SPAD502 e emissão da fluorescência em porta-enxertos de citros. Revista Ceres, 51(294), 1-9.

Banzatto, D. A., \& Kronka, S. N. (2013). Experimentação agrícola (4. ed., 237 pp.). Jaboticabal: Funep.

Barbosa, C. J., \& Rodrigues, A. S. (2015). Citrus tristeza. Revista Brasileira de Fruticultura, 36(3), 525-770.

Brasil. Ministério da Agricultura, Pecuária e Abastecimento - MAPA. (2009). Regras para análise de sementes (399 pp.). Brasília: MAPA.

Carvalho, J. A., Pinho, E., Oliveira, J. Á., Guimarães, R. M., \& Bonome, L. (2002). Qualidade de sementes de limãocravo (Citrus limonia Osbeck) durante o armazenamento. Revista Brasileira de Sementes, 24(1), 286-298.

Carvalho, L. M., Carvalho, H. W. L., Soares Filho, W. S., Martins, C. R., \& Passos, O. S. (2016). Porta-enxertos promissores, alternativos ao limoeiro 'Cravo', nos Tabuleiros Costeiros de Sergipe. Pesquisa Agropecuária Brasileira, 51(2), 132-141.

Coordenadoria de Defesa Agropecuária do Estado de São Paulo - CDA. (2018). Dados da citricultura do Estado de São Paulo por variedade base: $1^{\circ}$ semestre 2017 (23 pp.). Campinas: CDA.

Cunha Sobrinho, A. P., Magalhães, A. F. J., Souza, A. S., Passos, O. S., \& Soares Filho, W. S. (2013). Cultura do Citros (vol. 1, 399 pp.). Brasília: Embrapa Informação Tecnológica.
Demba, W. G. P. (2013). Conservação de sementes de porta-enxertos de citros em função dos teores de água das sementes e do ambiente de armazenamento (Dissertação de Mestrado). Universidade Federal de Viçosa, Viçosa.

Duarte, F. E. V. O., Barros, D. R., Girardi, E. A., Soares Filho, W. S., \& Passos, O. S. (2013). Polyembryony and morphological seed traits in citrus rootstocks. Revista Brasileira de Fruticultura, 35(1), 246-254.

Eiras, M., Silva, S. R., Stuchi, E. S., Targon, M. L. P. N., \& Carvalho, S. A. (2009). Viroids in citrus. Tropical Plant Pathology, 34(5), 275-296.

Ferreira, D. F. (2014). Sisvar: a guide for its Bootstrap procedures in multiple comparisons. Ciência e Agrotecnologia, 38(2), 109-112.

Fochesato, M. L., Souza, P. V. D., Schafer, G., \& Maciel, H. S. (2006). Produção de mudas cítricas em diferentes porta-enxertos e substratos comerciais. Ciência Rural, 36(5), 1397-1403.

Giuliani, J. C., Rieth, S., Soares, W., Lourosa, G. V., $\&$ Souza, P. V. D. (2014). Substratos e recipientes para a produção de porta-enxertos de citros irrigados por subcapilaridade. Ciência Rural, 44(3), 446-452.

Hong, T. D., \& Ellis, R. H. (1995). Interspecific variation in seed storage behavior within two genera - coffea and citrus. Seed Science and Technology, 23(1), 165-181.

King, M. W., \& Roberts, E. H. (1979). The storage of recalcitrant seeds: achievements and possible approaches (22 pp.). Rome: IBPGR.

Montenegro, H. W. S., \& Salibe, A. A. (1960). Conservação de sementes de porta enxertos para Citrus. Revista de Agricultura, 35(2), 109-135.

Najar, A., Hamdi, I., \& Ben, M. (2018). Citrus viroids: characterization, prevalence, distribution and struggle methods. Journal of New Sciences, 50(1), 3129-3137.

Nakagawa, J. (1994). Testes de vigor baseados no crescimento de plântulas. In R. D. Vieira, \& N. M. Carvalho. Testes de vigor em sementes (164 pp.). Jaboticabal: Funep.

Passos, O. S. (2012) Citrandarins: os porta-enxertos Indio, Riverside e San Diego (3 pp.). Cruz da Almas: Embrapa Mandioca e Fruticultura.

Passos, O. S., Soares Filho, W. S., \& Cunha Sobrinho, A. P. (2011a) Citrandarin 'Índio': nova opção de portaenxerto para a citricultura brasileira (2 pp.). Cruz da Almas: Embrapa Mandioca e Fruticultura. 
Passos, O. S., Soares Filho, W. S., \& Cunha Sobrinho, A. P. (2011b) Citrandarin 'Riverside': nova opção de porta-enxerto para a citricultura brasileira (2 pp.). Cruz da Almas: Embrapa Mandioca e Fruticultura.

Passos, O. S., Soares Filho, W. S., \& Cunha Sobrinho, A. P. (2011c) Citrandarin 'San Diego': nova opção de porta-enxerto para a citricultura brasileira (2 pp.). Cruz da Almas: Embrapa Mandioca e Fruticultura.

Passos, O. S., Soares Filho, W. S., Cunha Sobrinho, A. P., Souza, A. S., Santos, L. C., \& Peixouto, L. S. (2007). Banco ativo de germoplasma de citros da Embrapa Mandioca e Fruticultura Tropical: passado, presente e futuro (Embrapa Mandioca e Fruticultura Tropical. Documentos, 163, 61 pp.). Cruz da Almas: Embrapa.

Pompeu Junior, J. P., \& Blumer, S. (2014). Híbridos de trifoliata como porta-enxertos para laranjeira Pêra. Pesquisa Agropecuária Tropical, 44(1), 10-1590.

Rodrigues, M. J. S., Girardi, E. A., Ledo, C. A. S., Santos, M. G., Passos, O. S., \& Soares Filho, W. S. (2012). Emergência de genótipos de citros com potencial de uso como porta-enxertos. In Anais do $22^{\circ}$ Congresso Brasileiro de Fruticultura (p. 5439). Bento Gonçalves: SBF.

Rodrigues, M. J. S., Oliveira, E. R. M., Girardi, E. A., Silva Ledo, C. A., \& Soares Filho, W. S. (2015). Fruit characterization and propagation of hybrid citrus rootstocks in protected environment. Revista Brasileira de Fruticultura, 37(2), 457-470.

Simonetti, L. M. (2015). Avaliação de novos híbridos de porta-enxertos para a laranjeira 'Valência' (Dissertação de Mestrado). Universidade Estadual Paulista "Júlio de Mesquita Filho", Botucatu.

Siqueira, D. L., Vasconcellos, J. F. F., Dias, D. C. F. S., \& Pereira, W. E. (2002). Germinação de sementes de porta-enxertos de Citros após o armazenamento em ambiente refrigerado. Revista Brasileira de Fruticultura, 24(2), 317-322.

Soares Filho, W. S., Cunha Sobrinho, A. P., \& Passos, O. S. (2003a). Limoeiro 'Cravo Santa Cruz': variedade com maior número de sementes (2 pp.). Cruz da Almas: Embrapa Mandioca e Fruticultura.

Soares Filho, W. S., Moreira, C. S., Cunha, M.A. P., Cunha Sobrinho, A. P., \& Passos, O. S. (2000). Poliembrionia e frequência de híbridos em Citrus spp. Pesquisa Agropecuária Brasileira, 35(4), 857-864.

Soares Filho, W. S., Vilarinhos, A. D., Alves, A. A. C., Cunha Sobrinho, A. P., Oliveira, A. A. R., Souza, A. S., Ledo, C. A. S., Cruz, J. L., Souza, L. D., Castro Neto, M. T., Guerra Filho, M. S., Passos, O. S., \& Meissner Filho, P. E. (2003b). Programa de melhoramento genético de citros da Embrapa mandioca e fruticultura: obtenção de híbridos. (Embrapa Mandioca e Fruticultura. Documentos, 106, 37 pp.). Cruz das Almas: Embrapa.

Soares, L. A. A., Brito, M. E. B., Fernandes, P. D., Lima, G. S., Soares Filho, W. S., \& Oliveira, E. S. (2015). Crescimento de combinações copa-porta-enxerto de citros sob estresse hídrico em casa de vegetação. Revista Brasileira de Engenharia Agrícola e Ambiental, 19(3), 211-217.

Sombra, K. E. S., Araújo, L. T. L., Santos Filho, L. G., Silva, J. A. N., \& Bastos, D. C. (2015). Emergência de plântulas de seis genótipos de porta- enxertos de citros. Anais do X Congresso Regional da Sociedade Brasileira de Economia, Administração e Sociologia Rural no Nordeste (p. 139). Arapiraca: SOBER.

Struiving, T. B., Machado, D. L. M., Santos, D., Siqueira, D. L., Lucena, C. C., \& Matarazzo, P. H. M. (2013). Qualidade fisiológica de sementes de citros durante o armazenamento em ambiente refrigerado. Ciência Rural, 43(10), 1777-1782.

Recebido: Julho 13, 2018 Aceito: março 07, 2019

Como citar: Sombra, K. E. S., Silva, A. C. C., Loureiro, F. L. C., Andrade, H. M., Bastos, D. C., \& Uchôa, C. N. (2019). Emergência e desenvolvimento inicial de porta-enxertos de citros no semiárido do Ceará, Brasil. Citrus Research \& Technology, v. 40, e1042. http://dx.doi.org/10.4322/crt.00162 